Conclusions Our findings point to some possible causes of the variation and lack of transparency in disability evaluation. More formal and explicit approaches to a professional consensus informed by up-to-date research findings would increase the trust in the final judgments. Measures to reduce the unwanted variation need to be tested in further research.

\section{THE OCUPATIONAL HEALTH INVESTIGATION OF GRADUATE PROGRAMS IN COLOMBIA: TOPICS AND CHALLENGES}

C P Beltran Bocanegra, Riveros Alvarez, Barrero Solano. Pontificia Universidad Javeriana, Bogota, Colombia

\subsection{6/oemed-2013-101717.25}

Objective Human talent is a major component of the coordinated action that is needed to conduct preventive efforts in Occupational Health. While a large number of graduate programs in Occupational Health have been recently created in Colombia; little is known about the knowledge that is being developed out of those programs. This study aimed to assess the contribution that recent thesis works have made to the Occupational Health field in Colombia.

Methods We attempted to identify and systematically describe by major topic, assessed risk factors, occupational health disease of interest and features of the study design all the thesis works produced during the last 8 years in accredited programs in the country.

Results We identified 466 works from 6 academic institutions. However, we could find information for only 355 of those works. Most of the studies were found to have medium quality. Most studies tried to evaluate working conditions (84.5\%) and were observational field studies (70.1\%); however, the employed evaluation criteria were typically missing in the reports. Also, there were a large number of reviews (18.7\%). The most commonly investigated area was ergonomics (55.3\%); and within that area, the most commonly analysed topic was the psychosocial risk factors and the risks associated with physical demands. Accordingly, the most attention was given to musculoskeletal disorders. The general topic with the least number of contributions was Occupational Medicine (6.7\%).

Conclusions This distribution of thesis works generally corresponds with the needs of the country based on the official country's prevalence of occupational disease. Nevertheless, we recommend that more efforts are dedicated to study vulnerable populations in the informal sector that makes up most of the working population in the country and are not subjected to official recording. Also, efforts should be made so that academic programs define minimum thesis' scope and quality requirements.

\section{Session: D. Exposure assessment II}

\section{NATIONWIDE MACHINE SAFETY INITIATIVE FOR SMALL METAL FABRICATION BUSINESSES}

${ }^{1} S$ Yamin, ${ }^{1}$ Parker, ${ }^{2}$ Brosseau, ${ }^{3}$ Gordon, ${ }^{1}$ Bejan, ${ }^{1}$ Skan. ${ }^{1}$ Park Nicollet Institute, St. Louis Park, United States of America; ${ }^{2}$ University of Minnesota, Minneapolis, United States of America; ${ }^{3}$ Interrobang Group, Minneapolis, United States of America

\subsection{6/oemed-2013-101717.26}

Machine-related injuries are a persistent risk for workers in fabricated metal products manufacturing. Smaller establishments are of particular concern because these firms typically have limited access to safety and health resources. A research partnership with worker's compensation insurers was created to develop, implement, and evaluate a program to help small $(<150$ employees) metal fabrication businesses prevent machine-related injuries. The National Machine Guarding Program is a widely applicable, sustainable machine safety intervention being delivered on-site to firms throughout the US.

A fundamental objective of this intervention is to assist metalworking firms with interpretation and implementation of applicable Occupational Safety and Health Administration (OSHA) and American National Standards Institute (ANSI) standards. Beyond this, the partnership has developed methods and materials to help small businesses establish their own programs and policies within four topic areas: safety leadership, lockout/tagout, job hazard analysis, and machine safeguarding.

Each participating business will receive four on-site visits: a baseline safety evaluation, intervention delivery visits at 3months and 6-months, and a follow-up safety evaluation at 12 months. Insurance safety consultants serve as field staff for this program and receive extensive training to perform machine safety audits and deliver the intervention.

Technical checklists are used to evaluate 26 different types of metal fabrication machinery. Checklist data are entered into software developed for this project. The software compiles category scores from all checklist responses into a concise report that is provided to each business.

The presentation will provide attendees with an understanding of the design, implementation, and evaluation of a computerised safety management system for metal fabrication industries. Preliminary results will be presented as a summary of baseline machine safety data collected at participating businesses during 2012-2013.

\section{SICK BUILDING SYNDROME IN PUBLIC SERVICES OF EURE-ET-LOIR'S DISTRICT, FRANCE}

${ }^{1} \mathrm{M}$ Riviere, ${ }^{2}$ Dorcas Lafitte, ${ }^{3}$ Gayral, ${ }^{2}$ Chaventré, ${ }^{3}$ Brachet, ${ }^{2}$ Ramalho, 'Jeannel. ${ }^{1}$ InVS, Orléans, France; ' 2 Centre scientifique et technique du bātiment, Paris, France; ${ }^{3}$ Delegation territoriale de I'Eure-et-Loir, ARS Centre, Chartres, France

10.1136/oemed-2013-101717.27

In September 2010, a sick building syndrome occurred after the relocation of three public services of Eure-et-Loir's district, France, in a renovated building in which foul odours have been perceived.

The establishment of a coordination unit allowed an interdisciplinary approach including clinical, epidemiological, psychosocial and environmental investigations. Concerning the epidemiological field, a transversal study has been realised by administrating a questionnaire to volunteered officers ( $n=48$ out of 54). The study period was from september 2010 to september 2011. Univariate and multivariate analysis allowed studying risk factors for symptoms apparition.

During the study period, $84 \%$ of the officers reported symptoms. Those were mainly distributed into three types: irritatives (63\%), general (51\%) and ears nose and throat symptoms (27\%). Multivariate analysis showed that: agents affected by irritative symptoms were originally in good health $(\mathrm{OR}=20,32$; [2.5-162.0]; the risk for all symptoms increased with the rise of the number of hours at work $(\mathrm{OR}=[1.1-1.4]$ and for workers in offices with old floor lining $(\mathrm{OR}=22,0[1.8-265.5]$. The building had an inappropriate ventilation system for a tertiary 